\title{
Role of Nursing in HSCT
}

\author{
Aleksandra Babic and John Murray
}

\subsection{Introduction: HSCT Nursing}

With the progress of HSCT in the early 1960s, it became clear that nurses play a crucial role within the multidisciplinary team (MDT) caring for patients and their families undergoing this intense treatment. The distress during the time prior to undergoing HSCT, during isolation, in the recovery phase and the time after (long-term recovery) is not to be underestimated.

The best compliment towards nursing was made by Prof. Edward Donnall Thomas, the 1990 Nobel Prize winner in Medicine who stated that 'nurses and nursing are my secret weapon without whom I could not have achieved my goals' (Appelbaum 2013).

Continuity of care is vital to patient's right from their initial attendance in hospital. Nurses are an advocate throughout the transplant and often act as a motivating force, supporting and advising as well as supplying physical, psychological and emotional care whilst patient's transition from acute care to long-term followup clinics. Experienced nurses with high levels

\footnotetext{
A. Babic $(\bowtie)$

IOSI-Istituto Oncologico della Svizzera Italiana, OSG, Bellinzona, Switzerland e-mail: Aleksandra.Babic@eoc.ch

J. Murray

Haematology and Transplant Unit,
}

The Christie NHS Foundation Trust, Manchester, UK of technical competencies offer patients and families excellent care and support in this challenging area.

Patient preparation for HSCT involves the use of chemotherapy and/or radiotherapy to eradicate the underlying disease of the patient. Throughout the transplant procedure, the patient needs special care to overcome the complications associated with treatment. Nurses must be aware of the possible complications in order to play a role in prevention or early detection of alarming signs, such as sepsis, fluid overload and organ dysfunction, taking appropriate measures to minimize adverse effects and restore the clinical balance of the patient. This care is very complex and requires a high level of skill (Wallhut and Quinn 2017).

The field of nursing research in HSCT has evolved from reflecting on symptom management and service development to quality of life and long-term survival topics. The FACT-JACIE International Standards Accreditation requires that the clinical programme has access to personnel who are formally trained, experienced and competent in the management of patients receiving cellular therapy (JACIE 7 th edition n.d.). Thus, it is important that training and competency programmes are structured and ongoing, with documented evidence of training topics and dates (Babic 2015). 


\subsection{Role of Nursing Throughout HSCT Patient Pathway}

HSCT is a standard therapy in a number of malignant and non-malignant conditions.

Pre-transplant assessments must be undertaken, and the results of these along with suitable donor medical clearance and cell availability are essential to ascertain that transplant is a valid option and can proceed safely.

Nurses are pivotal in implementing practices to prevent and manage infections and other serious effects following HSCT (Sureda et al. 2015; Kenyon and Babic 2018) as:

- Bleeding risk caused by thrombocytopenia

- Tiredness and fatigue caused by the decreased haemoglobin levels and lasting effects of chemo-/radiotherapy and associated medications

- Pain due to mucositis

- Sepsis

- Reduced nutrition

- Psychosocial distress

- Isolation

\subsubsection{The Role of the Transplant Coordinator (TC)}

Many transplant coordinators are nurse specialists who focus their role on the individual needs of the patient and families; however, some centres have medical staff that organize transplants. $\mathrm{TC}$ is the person who should:

- Ensure that timely events occur for each patient and their families undergoing HSCT and that the patients are physically and psychologically prepared for the treatment.

- Provide a high level of care and management, inform and educate the patient, have holistic knowledge of the patient, participate in specific or advanced nursing practices (bone marrow sampling, HLA typing, transplant recipient care) and coordinate all the transplant logistics.
- Ensure that a suitable source of cells is available following the high-dose chemotherapy or immunosuppressive treatment that the patient will receive. Make requests to donor search panels, and order cells once the ideal match has been identified by the transplant physician.

- Support the patient with verbal and written information, and educate them about the whole process from typing to transplant. The TC will coordinate all of the care and embodies a clinical nursing function where emphasis is placed on specialization in a clearly defined area of care.

- Actively participate in the JACIE process of accreditation of transplant centres by writing and evaluating SOPs and being a valued member of the MDT and ward team offering teaching and advice.

\subsection{Specific Aspects with a Prominent Role for Nurses}

\subsubsection{Venous Access Device (VAD)}

Education and training should not be limited to the care and maintenance after insertion of the VAD but should be focused on well-being and patient safety. An algorithm for choosing the right VAD for the right patient should start with the diagnosis and treatment plan. The best VAD should be chosen based on the $\mathrm{pH}$ and osmolarity of the drugs used during the whole treatment period and the vein condition and should include the option for (partial) home infusion treatment.

Within the range of CVAD (central VAD), a peripherally inserted central catheter (PICC) is seen frequently in haematology patients, often as an alternative for a tunnelled CICC (centrally inserted central catheter) such as a Hickman catheter (see Chap. 22).

Nurses are responsible for the safe administration of drugs such as chemotherapy, IS immunosuppressive drugs and blood products as well as 
parenteral nutrition and symptom control drugs. The accurate handling and taking care of the central venous catheter and infusion pump systems are vital in the process because the catheter is related to the highest risk of infections. The use of an Aseptic Non Touch Technique (ANTT) (Pratt et al. 2007) and its ten principles of care have led to a decrease in catheter-related infections.

GAVeCeLT (Gli Accessi Venosi Centrali a Lungo Termine) (Pittiruti and Scoppettuolo 2017) has developed an algorithm for the choice of the most appropriate VAD, based on the best evidence available in the international guidelines, the bundle for the safe implantation of PICCs (see Table 32.1).

Table 32.1 The bundle for the safe implantation of $\mathrm{PICCs}^{\mathrm{a}}$

\section{The goals of the bundle are to minimize}

1. Complications related to venipuncture: failure, repeated punctures, nerve injury, arterial injury

2. Malposition

3. Venous thrombosis

4. Dislocation

5. Infection

In order to reach the goal, the SIP protocol was developed and needs to be followed

1. Bilateral US scan of all veins at the arm and neck

2. Handwashing, aseptic technique and maximal barrier protection

3. Choice of the appropriate vein at the midarm (vein $\mathrm{mm}=$ or $>$ cath Fr)

4. Clear identification of median nerve and brachial artery

5. Ultrasound-guided venipuncture

6. US tip navigation during introduction of the PICC

7. Electrocardiography method for assessing tip position

8. Securing the PICC with cyanoacrylate glue, sutureless devise and transparent dressing

Infections in PICCs to be close to zero if a bundle of preventive measures are taken ${ }^{\mathrm{b}}$

- Site selection

- Skin disinfection with $2 \%$ chlorhexidine in $70 \%$ gluconate

- Hand hygiene

- Maximum barrier precautions

- Daily control on indication and on complications

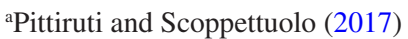

${ }^{\mathrm{b}}$ Harnage (2012)

\subsubsection{Early and Acute Complications}

They occur following transplantation when the patient has reduced tolerance due to neutropenia and/or increased intestinal permeability. In neutropenia, the number of white blood cells decreases significantly, resulting in aplasia with an increased risk of infection. An increased permeability of the intestinal wall is caused by intensive chemotherapy damaging the gastrointestinal mucosa. As a result, pathogenic bacteria (bodily bacteria or bacteria from the diet) can enter the bloodstream and cause sepsis.

Early complications generally occur within 100 days post HSCT. In the early phase of HSCT, the main risk factors for infections are neutropenia-barrier breakdown due to mucositis, indwelling catheters, depressed T-cell and B-cell function and aGvHD.

Two of the most common early complications are oral mucositis and sepsis. Some other relatively rare complications are HC, ES, IPS and DAH. TAM and SOS/VOD are analysed in Chaps. 42, 49 and 50. For all complications there are locally agreed recommendations for prevention and principles for nursing care, with monitoring and prompt intervention that may have an influence on patients' morbidity and mortality.

\subsubsection{Oral Mucositis (OM)}

Oral mucositis (OM) has been defined (Rubenstein et al. 2004) as the inflammation of the mucosal membrane, characterized by ulceration, which may result in pain, swallowing difficulties and impairment of the ability to talk. The mucosal injury caused by OM provides an opportunity for infection to flourish and in particular putting the severely immunocompromised patient in the HSCT setting at risk of sepsis and septicaemia.

OM and oral problems in the HSCT setting can be expected to occur in as many as $68 \%$ of patients undergoing autologous HSCT and 98\% of patients undergoing allogeneic HSCT (EORTC Guidelines). With the increasing use of targeted drug therapies and approaches in the cancer and 
haematology setting, problems in the oral cavity will increase and become even more of a challenge (Quinn et al. 2015).

All treatment strategies aimed at improving mouth care are dependent on four key principles: accurate assessment of the oral cavity, individualized plan of care, initiating timely preventative measures and correct treatment (Quinn et al. 2008). The assessment process should begin prior to HSCT by identifying all the patient risks most likely to increase oral damage.

The choice of prevention regimens should be guided by evidence based on expert opinion interventions, working with the patient to reduce their potential risk of oral mucositis occurring.

All treatment plans should be based upon the grading of oral damage and patient reports, and these may include the use of topical analgesics and the use of opiates (Elad et al. 2015).

\subsubsection{Sepsis}

Sepsis is a life-threatening condition caused by aberrant and dysregulated host response to infection (Elad et al. 2015). The most important action to prevent infections acquired by exogenous organisms is good hand hygiene performed correctly (Hand Hygiene Guidelines). Appropriate clean work clothes, with short sleeves, no jewellery and no neck tie are the responsibility of all staff. Protective isolation during the neutropenic phase is recommended, and the patient should not be in contact with any staff or visitors with symptoms of infection. For prevention of endogenous infections, oral hygiene and skin care to maintain the mucosal and skin barrier and use of prophylactic antibiotics are the most important actions. Correct handling of any indwelling catheters is also a key nursing responsibility in infection control.

Other areas where infections can be prevented are air and water quality, food hygiene and environmental cleaning. Environmental cleaning includes medical equipment as well.

Early recognition and treatment are vital for a successful outcome of sepsis. Temperature, pulse, blood pressure, respirations and saturation (vital signs) should be frequently monitored.
Signs of infection are not always obvious, but if the patient has a temperature $\geq 38.0^{\circ} \mathrm{C}$, cultures should be taken, IV antibiotics and IV fluids started or increased and oxygen therapy initiated.

The goal is always to start antibiotic treatment within $1 \mathrm{~h}$ from detection of fever and is the most critical period in the patient's survival from sepsis. Early recognition and intervention are achieved by frequent monitoring of the patient's vital signs and general condition and paying attention to subtle changes that should be promptly reported, such as mental state alteration or mottled skin.

Alert for immediate action are when a previously well patient only responds to pain or becomes unresponsive, becomes confused and has a systolic blood pressure of $<90 \mathrm{mmHg}$ or a fall of $>40 \mathrm{mmHg}$ from baseline; an elevated heart rate $>130 \mathrm{bpm}$; a respiratory rate of $>25$ per min, requiring oxygen to maintain saturations $>92 \%$; a non-blanching rash or mottled, ashen or cyanotic skin, not passed urine in the last $18 \mathrm{~h}$; an output of $<0.5 \mathrm{ml} / \mathrm{kg} / \mathrm{h}$; a lactate of $>2 \mathrm{mmol} / \mathrm{l}$; or received recent chemotherapy.

Immediate action is required at the first indication of sepsis. The concept of the sepsis six and the severe sepsis resuscitation bundle (Daniels et al. 2011) has been developed as a guide to prioritize interventions in patients where sepsis is suspected:

1. Oxygen therapy aims to keep saturations $>94 \%$ (88-92\% if at risk of $\mathrm{CO}_{2}$ retention, e.g. COPD).

2. Blood cultures, at least a peripheral set, consider CSF, urine, sputum, chest X-ray and urinalysis.

3. IV antibiotics, according to hospital policy, consider allergies prior to administration.

4. Fluid resuscitation, if hypotensive or lactate $>2 \mathrm{mmol} / \mathrm{l}, 500 \mathrm{ml}$ bolus stat, may be repeated if clinically indicated. Do not exceed $30 \mathrm{ml} /$ $\mathrm{kg}$.

5. Serial serum lactates corroborate high VBG lactate with arterial sample. If lactate $>4 \mathrm{mmol} / \mathrm{l}$, call critical care for support. Recheck after each $10 \mathrm{ml} / \mathrm{kg}$ challenge. 
6. Assess urine output which may require catheterization, and ensure fluid balance chart commenced and completed hourly.

When treatment has been initiated, the patient must be continually monitored to determine the effect of treatment or worsening of the condition. This includes vital signs, fluid balance including weight and assessment of identified and/or potential infection sites (mouth, skin, all indwelling catheters, urine, stools, etc.), mental status, signs of bleeding, pain and general appearance and well-being.

Antibiotics should be delivered with strict adherence to the prescribed time schedule. Antipyretic agents should be avoided since they may mask fever but may under certain circumstances be used to alleviate patient discomfort and pain.

\subsubsection{Pain}

Pain in the HSCT setting is most commonly experienced as a result of mucositis, but patients will also report other pain such as bone pain associated with G-CSF, abdominal pain due to diarrhoea or general discomfort with fluid accumulation. A comprehensive evaluation of the pain, location, characteristics, onset, duration, frequency and severity, exacerbating and relieving factors, should be included. This assessment should be supported by the patient's non-verbal reactions such as facial expression, pallor, tempo of speech, body position, etc. as well as their vital signs.

\subsubsection{GvHD}

GvHD remains a leading cause of non-relapse mortality and is associated with a high morbidity that increasingly affects quality of life (Lee et al. 2003; Dignan 2012). Nursing care of patients with GvHD is highly complex and extremely stressful especially in the acute setting in patients with grades 3-4 skin and GI involvement (Table 32.2). Supportive nursing care to complement medical interventions aims to offer symptomatic comfort and relief.

There are many manifestations of GvHD, and nurses are able to advise patients with respect to many of these including eye, mouth and genital care. Further readings: GvHD chapter in the
Table 32.2 Nursing care of patients with skin and gastrointestinal GvHD

\section{Skin care}

1. Maintain integrity of the skin; regular application of cream, ointment or gel; patient choice of vehicle

2. Emollient application, high or low water content to be considered, QV, hydromol or diprobase

3. At least 30-min gap between emollient and steroid cream applications

4. Topical steroids, strength decided by site and length of treatment

5. Menthol cream for painful and pruritic skin, cooling effect

6. Use high-factor sunscreen SPF 50+

7. Always apply creams to make the skin appear shiny; adult body will require $500 \mathrm{~g}$ per week

8. Apply in one direction, direction of hair growth, do not scrub on

9. Medical grade silk clothing

10. Good fluid intake and nutrition

11. Organic coconut oil or other natural lipids

12. Aloe vera gels; do not use alone as they will dry the skin

Gastrointestinal

1. Ensure stool samples are taken to exclude infection

2. Adequate oral intake with strict fluid balance

3. Small and frequent high-calorie food and drinks

4. Antiemetics

5. Loperamide, codeine and octreotide may be used to stem diarrhoea

6. Rest bowel and use parenteral feeding

7. Consider the use of radiologically inserted gastrostomy (RIG) feeding

8. Flexi-seal faecal collection device

EBMT Textbook for nurses 2018 (Kenyon and Babic 2018).

\subsubsection{Long-Term Complications and Side Effects Post Allo-HCST}

Long-term side effects after allo-HSCT include non-malignant organ or tissue dysfunction, changes in quality of life, infections related to abnormal immune reconstitution and secondary cancers. Many of these can be attributed to effects of chronic graft-versus-host disease (Dignan 2012; Bhatia 2011; Mohty and Mohty 2011). With advances achieved in terms of supportive care, it is reasonable to expect outcomes to improve steadily, and consequently increasing numbers of transplant survivors will be facing life after the initial 
transplant experience. For some survivors the burden of long-term morbidity is substantial, and long-term follow-up of patients who received alloHSCT is now widely recommended.

\section{Key Points}

- Specific technical care activities require nursing knowledge and specific skills in the field of HSCT such as instrument manipulation, knowledge of technologies and the use of special protocols to effectively intervene in complex situations and deal with acute and chronic HSCT complications.

- As patients become more complex, so does the care that they require.

- It is essential that nursing adapts to these challenges and improves in both the quality and expertise that is vital to improve patient survival and overall experience of this life-changing treatment.

- The predominant role for nurses is focused to vascular access device, oral mucositis and other early complications as HC, ES, IPS and DAH. TAM and SOS/VOD, sepsis, pain, GVHD and several late complications.

\section{References}

Appelbaum FR. 2013. http://www.hematology.org/ Thehematologist/Profiles/1088.aspx.

Babic A. Transplant unit personnel competency maintenance: online testing by sharepoint application. EBMT 2015. Oral presentation N003.

Bhatia S. Long-term health impacts of hematopoietic stem cell transplantation inform recommendations for follow-up. 2011. https://www.ncbi.nlm.nih.gov/pmc/ articles/PMC3163085/.

Daniels R, Nutbeam T, McNamara G, Galvin C. The sepsis six and the severe sepsis resuscitation bundle: a prospective observational cohort study. Emerg Med J. 2011;28:507-12.

Dignan FL, Amrolia P, Clark A, Haemato-oncology Task Force of British Committee for Standards in
Haematology, British Society for Blood and Marrow Transplantation, et al. Diagnosis and management of chronic graft-versus-host disease. Br J Haematol. 2012;158:46-61.

Elad S, Raber-Durlacher JE, Brennan MT, et al. Basic oral care for hematology-oncology patients and hematopoietic stem cell transplantation recipients: a position paper from the joint task force of the Multinational Association of Supportive Care in Cancer/International Society of Oral Oncology (MASCC/ISOO) and the European Society for Blood and Marrow Transplantation (EBMT). Support Care Cancer. 2015;23:223-36.

Harnage S. Seven years of zero central-line-associated bloodstream infections. Br J Nurs. 2012;21:S6, S8, S10-2.

JACIE 7th Edition Standards. www.Jacie.org.

Kenyon M, Babic A. European blood and marrow transplantation textbook for nurses. Cham: Springer; 2018. isbn:978-3-319-50025-6.

Lee SJ, Vogelsang G, Flowers MED. Chronic graftversus-host disease. Biol Blood Marrow Transplant. 2003;9:215-33.

Mohty B, Mohty M. Long-term complications and side effects after allogeneic hematopoietic stem cell transplantation: an update. Blood Cancer J. 2011;1:e16.

Pittiruti M, Scoppettuolo G. The GAVeVeLT manual of PICC and midline, indications, insertion, management. St. Paul: Edra; 2017.

Pratt RJ, Pellowe CM, Wilson JA, et al. National evidencebased guidelines for preventing healthcare-associated infections in NHS hospitals in England. J Hosp Infect. 2007;65(Suppl 1):S1-64.

Quinn B, Potting C, Stone R, et al. Guidelines for the assessment of oral mucositis in adult chemotherapy, radiotherapy and haematopoietic stem cell transplant patients. Eur J Cancer. 2008;44:61-72.

Quinn B, Thompson M, Treleaven J, et al. United Kingdom oral care in cancer guidance. 2nd ed. 2015. www.ukomic.co.uk. Accessed 03 Sep 2016.

Rubenstein EB, Peterson DE, Schubert M, Mucositis Study Section of the Multinational Association for Supportive Care in Cancer, International Society for Oral Oncology, et al. Clinical practice guidelines for the prevention and treatment of cancer therapyinduced oral and gastrointestinal mucositis. Cancer. 2004;100(Suppl 9):2026-46.

Sureda A, Bader P, Cesaro S, et al. Indications for alloand auto-SCT for haematological diseases, solid tumours and immune disorders: current practice in Europe, 2015. Bone Marrow Transplant. 2015;50: 1037-56.

Wallhut E, Quinn B. Early and acute complications and the principles of HSCT nursing care. In: Wallhult E, Quinn B, Kenyon M, Babic A, editors. The European blood and marrow transplantation textbook for nurses. 2017. https://doi.org/10.1007/978-3-319-50026-3_9. 
Open Access This chapter is licensed under the terms of the Creative Commons Attribution 4.0 International License (http://creativecommons.org/licenses/by/4.0/), which permits use, sharing, adaptation, distribution and reproduction in any medium or format, as long as you give appropriate credit to the original author(s) and the source, provide a link to the Creative Commons license and indicate if changes were made.

The images or other third party material in this chapter are included in the chapter's Creative Commons license, unless indicated otherwise in a credit line to the material. If material is not included in the chapter's Creative Commons license and your intended use is not permitted by statutory regulation or exceeds the permitted use, you will need to obtain permission directly from the copyright holder.

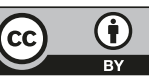

